\title{
Etudes histologiques et histochimiques de surrénales de Cobayes traités par des extraits ascaridiens
}

\author{
par M. BABONITS, F. WIENER, B. FAZAKAS et A. BEDÖ \\ Institutul de Medicină si Farmacie din Tirgu-Mures, Disciplina de Parazitologie \\ [Directeur : Fazakas Bela], str. Gh. Marinescu nr. 38, Tirgu Mures, Romania
}

\begin{abstract}
Résumé
Dans le présent travail les auteurs se sont proposé d'étudier les modifications histopathologiques et histophysiopathologiques des glandes surrénales, après l'action d'extrait ascaridien dans des conditions expérimentales.

L'action non spécifique de la substance administrée se manifeste par une hyperplasie corticale, par une activité augmentée de la phosphatase acide et surtout de la succinodeshydrogénase, ce qui pourrait être en rapport avec les nécessités énergétiques augmentées de la glande dans la production des corticostéroïdes. Les signes inflammatoires, les infarctus anémiques des régions de la zone réticulaire, associés à une accumulation excessive de glycogène dans cette zone de nécrobiose, reflètent l'action toxique directe de l'extrait ascaridien.
\end{abstract}

\section{Summary}

In the present work an attempt has been made to study the histopathological and histophysiopathological modifications of the suprarenal glands after effect of ascaridian extract in experimental conditions.

As a result of the non specific effect of the extract, we observe a cortical hyperplasia shown by an increased activity of the acid phosphatase and specially of the succinodeshydrogenase. This fact could be in relation with the increase of the energical needs of the gland in the production of corticosteroids. The signs of inflammation, the anemic infarcts in the region of the reticular zone, associated with an excessive accumulation of glycogen in this necrosis zone, reflect the direct toxic effect of the ascaridian extract. 


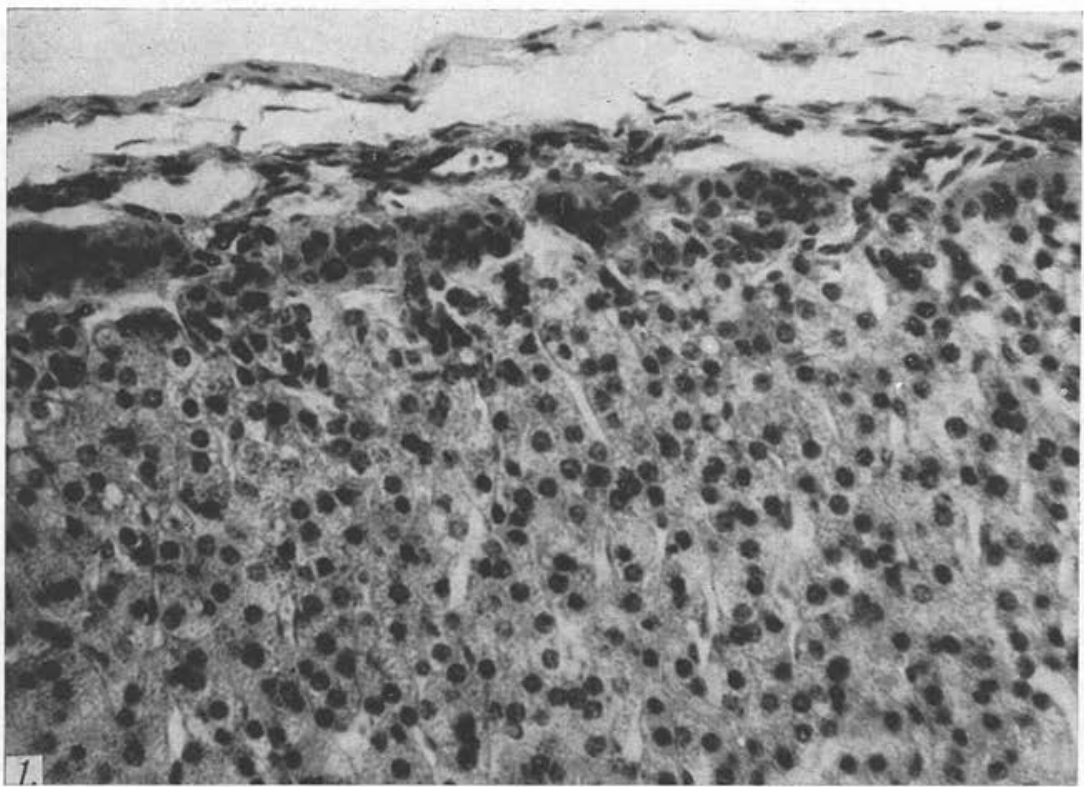

Fig. 1. - Surrénale. Animal témoin. Col. H.E.Oc. : F-1, Ob. : 20 x.

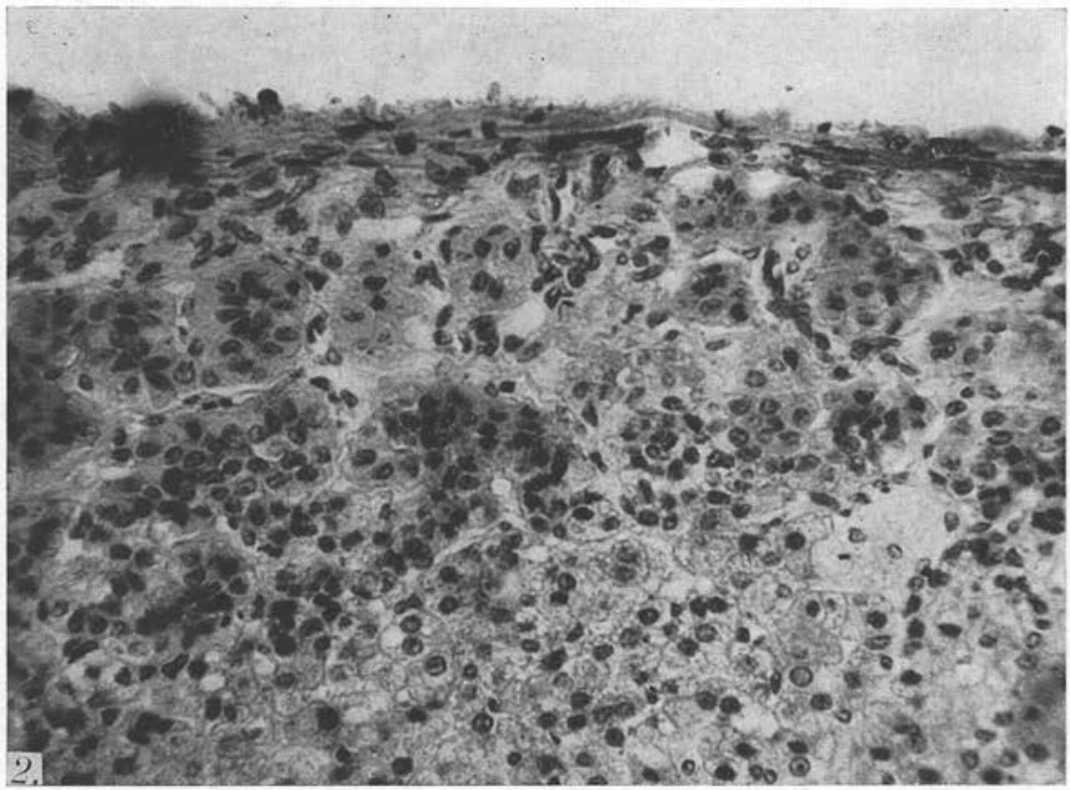

FIg. 2. - Surrénale. Animal traité avec de l'extrait ascaridien aqueux total.

On peut observer l'hyperplasie de la zone glomérulaire. Col. H.E.Oc. : F-1, Ob. : 20 x, 
Lors de nos travaux antérieurs, nous avons étudié l'influence de l'extrait ascaridien sur les différents organes, par des méthodes histologiques et histochimiques, en établissant les modifications significatives de l'activité de certains enzymes, ainsi que la localisation topochimique de certaines substances au niveau des organes étudiés (3). Dans le présent travail, nous nous sommes proposé d'étudier les modifications histopathologiques et histophysiologiques des glandes surrénales après action d'extrait ascaridien dans des conditions expérimentales, afin d'élucider d'une manière plus complexe le mécanisme d'action de ces substances.

\section{Matériel et méthodes.}

Les ANIMAUX D'EXPÉRIENCE ET LE MODE D'INOCULATION. - Quarante cobayes des deux sexes, ayant un poids moyen de 350-400 g ont été inoculés par voie intrapéritonéale avec $1 \mathrm{ml}$ d'extrait aqueux d'Ascaris lumbricoides, tous les deux jours pendant trente jours consécutivement.

Après la dernière inoculation, on a sacrifié les animaux par décapitation et on a fixé les glandes surrénales dans les solutions qui correspondent aux méthodes appliquées. En même temps, on a également fixé les surrénales des animaux témoins, inoculés par voie intrapéritonéale avec du sérum physiologique en quantité correspondante à l'extrait ascaridien administré aux animaux d'expérience.

Extrait AqueuX total. - La préparation de l'extrait ascaridien utilisé a été décrite dans un travail antérieur (3).

Méthodes histologiques et histochimiques. - Afin d'avoir une vue d'ensemble de structure histologique, les organes prélevés furent fixés dans une solution fixatrice Supiformeils, inclus dans la paraffine et colorés par l'hématoxyline-éosine et par la coloration trichromique Székely.

Pour mettre en évidence les acides nucléiques (ADN et ARN) du glycogène, des mucopolysaccharides acides et neutres, on a appliqué la coloration Feulgen, vert de méthyl-pyronine, chromalaun-gallocianique, P.A.S., bleu alcien et bleu de toluidine.

Les phosphatases alcaline et acide furent mises en évidence par la méthode Gomori. On a fixé les pièces en formol de $4 \%(\mathrm{Ph}: 7,2)$ pendant 12-16 heures à une température de $4^{\circ} \mathrm{C}$, en les sectionnant avec le microtome de congélation.

La succino-déhydrogénase a été mise en évidence par la méthode préconisée par Rutenburg et collab. (10). Les pièces ont été congelées à $-20^{\circ} \mathrm{C}$ et sectionnées au microtome à congélation, les sections ayant une épaisseur de 15-20 microns.

\section{Résultats.}

OBSERVATIONS histopathologiques. - La zone corticale des animaux traités présente une hyperplasie reflétée surtout par l'hypertrophie de la zone glomérulaire et fasciculaire (fig. 1 et 2 ). En même temps, on observe une hyperémie manifestée par la dilatation des vaisseaux corticaux qui sont très souvent obturés par des hématies. On observe également une migration des leucocytes des vaisseaux qui pénètrent dans les 


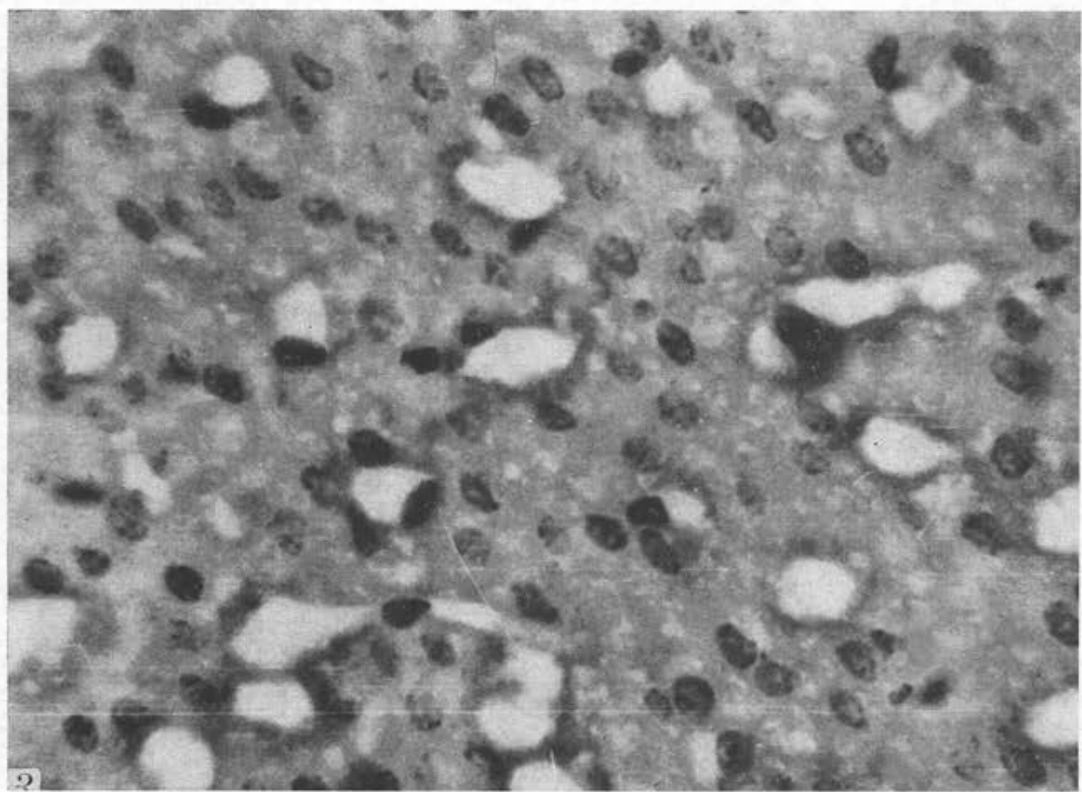

Fig. 3. - Surrénale. Animal témoin. La réaction Feulgen dans la zone réticulaire est normale. Oc. : F-1, Ob. : $40 \mathrm{x}$.

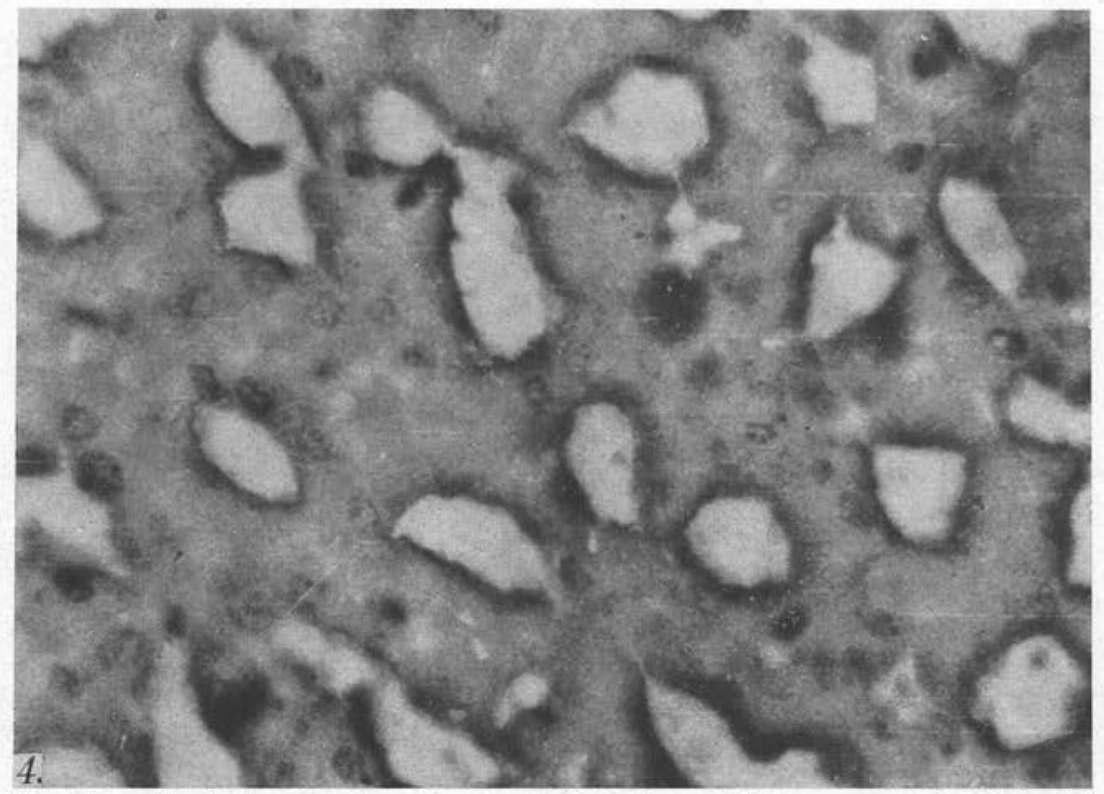

Fig. 4. - Surrénale. Animal traité avec de l'extrait ascaridien aqueux total. L'intensité de la réaction de Feulgen dans la zone réticulaire est diminuée, les noyaux présentent une diminution de la quantité de l'A.D.N. Oc. : F-1, Ob. : 40 x. 


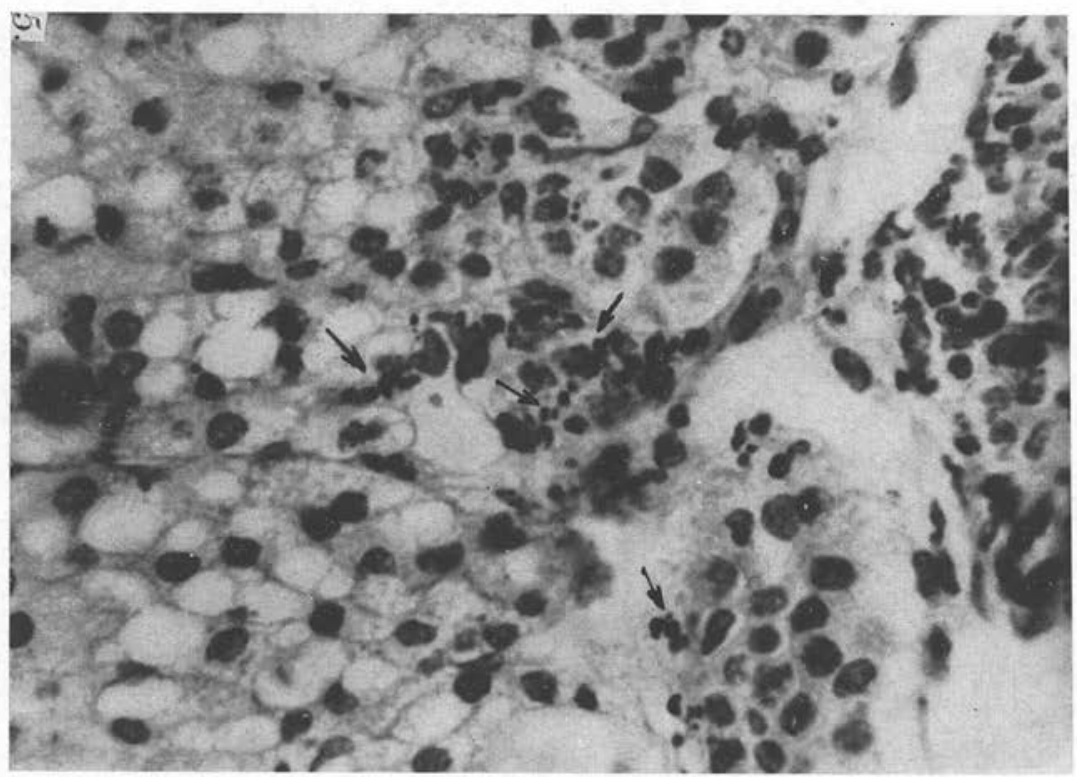

Fig. 5. - Surrénale. Glycogène. Animal traité avec de l'extrait ascaridien aqueux total. On peut observer dans la zone glomérulaire des granulations de glycogène qui se localisent dans les leucocytes extravasées, Oc.: F-1, Ob. : $40 \mathrm{x}$.

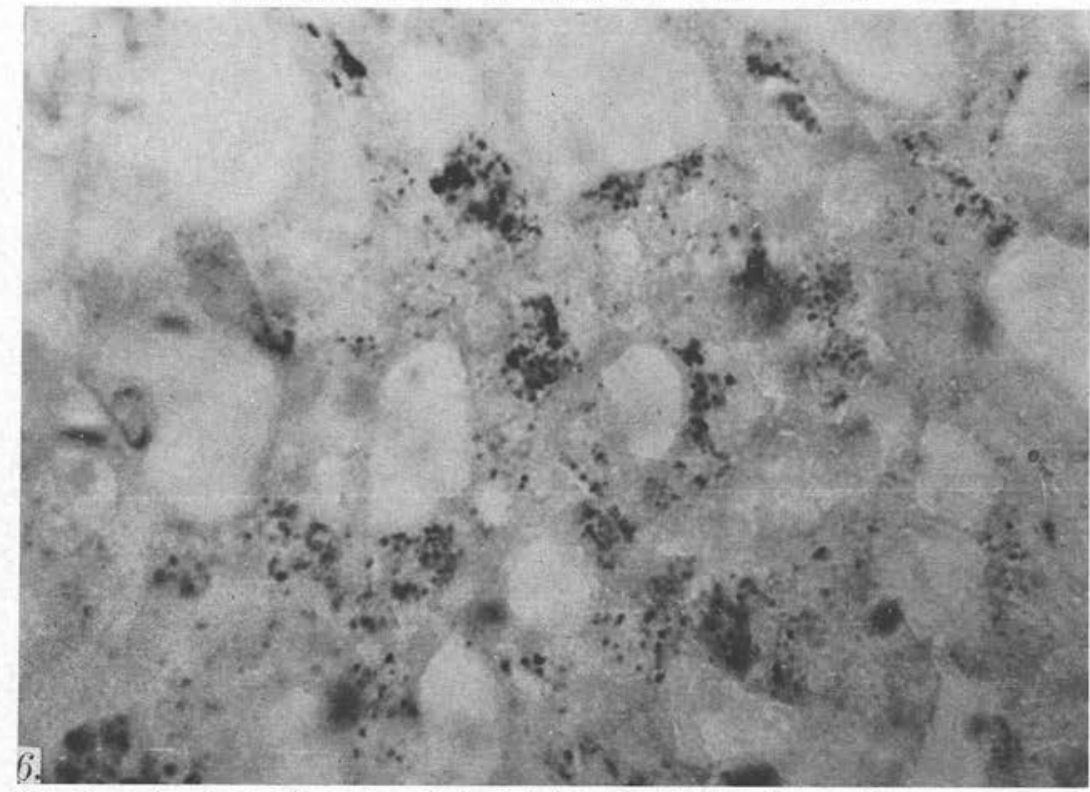

Fig. 6. - Surrénale. Glycogène. Animal traité avec de l'extrait ascaridien aqueux total. On peut observer l'accumulation de glycogène dans la zone réticulaire avec signes de nécrobiose. Réaction PAS. Oc. : F-1, Ob. : 40 x. 
différentes zones de la région corticale. Chez certains animaux traités, au niveau de la zone réticulaire, on observe dans les territoires délimités une picnose nucléaire jusqu'à la disparition complète des noyaux et de la structure caractéristique de cette zone, qui présente des signes évidents de nécrobiose.

Du point de vue histologique, la zone médullaire a gardé sa structure, sans présenter de modifications histologiques.

Acide desoxyribonucléique. - L'intensité de la réaction de Feulgen dans la zone réticulaire, qui correspond aux territoires dans lequel les noyaux sont picnotiques, est faible, ce qui dénote une réduction de la quantité d'ADN dans ces cellules gravement lésées. D'ailleurs l'ADN dans les autres zones ne présente pas de différences essentielles en comparaison avec celui des animaux témoins (fig. 3 et 4).

ACIDE RiBonuclérque. - La localisation topochimique de l'acide ribonucléique est identique avec celle chez les animaux témoins.

GLYCOGÈnE. - Dans la zone glomérulaire, l'on observe une répartition inégale des granulations de glycogène, qui se localisent dans les leucocytes extravasés ou se trouvent dispersés à l'état libre par la désintégration des leucocytes dans cette zone (fig. 5). Parfois, les granulations de glycogène se localisent aussi dans la zone fasciculaire toujours grâce à la présence des leucocytes dans ce territoire. Dans les glandes surrénales des animaux chez lesquels la zone réticulaire est gravement lésée, on observe une accumulation intensive de glycogène (fig. 6).

Mucopolysaccharides NeUtres. - On peut remarquer en comparaison avec les animaux témoins, la présence de substance P.A.S. positive dans la capsule des surrénales des animaux traités. Cette accumulation est plus intense autour et dans les parois vasculaires, ce qui traduit une certaine lésion de ces derniers sous l'influence de l'extrait ascaridien.

Mucopolysaccharides ACIDES. - La coloration au bleu alcien ne révèle pas de différences essentielles avec les animaux témoins, mais on peut cependant mentionner que la zone glomérulaire des animaux traités présente une coloration diffuse plus intense que la zone glomérulaire des animaux de contrôle. De même, la coloration au bleu de toluidine met en évidence dans les couches supérieures de la substance corticale un nombre relativement grand de cellules métachromatiques, probablement d'origine réticulaire. Dans la capsule glandulaire des animaux traités, le nombre de cellules métachromatiques est également plus grand que chez les animaux témoins.

Phosphatase alcaline. - La répartition topochimique de la phosphatase alcaline est identique avec celle des animaux de contrôle, étant localisée surtout dans la zone glomérulaire et dans la zone fasciculaire. En ce qui concerne l'activité de cet enzyme, celle-ci est plus réduite chez les animaux d'expérience que chez les animaux témoins, bien que la zone glomérulaire et la zone fasciculaire des surrénales des animaux traités soient hypertrophysées. 


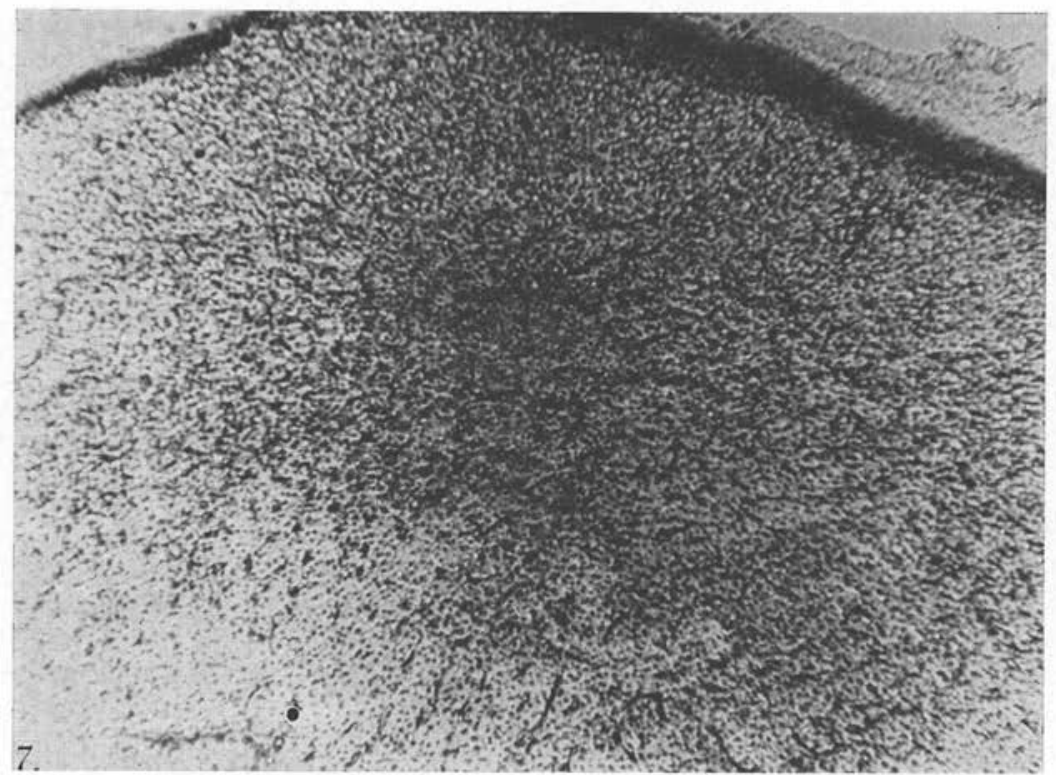

Fig. 7. - Surrénale. Animal Témoin. L'activité de la phosphatase acide s'est localisée dans la région corticale, plus intense dans la zone glomérulaire. Méthode Gomori. Oc. : 15 x, Ob. : Lupe 4,5/30

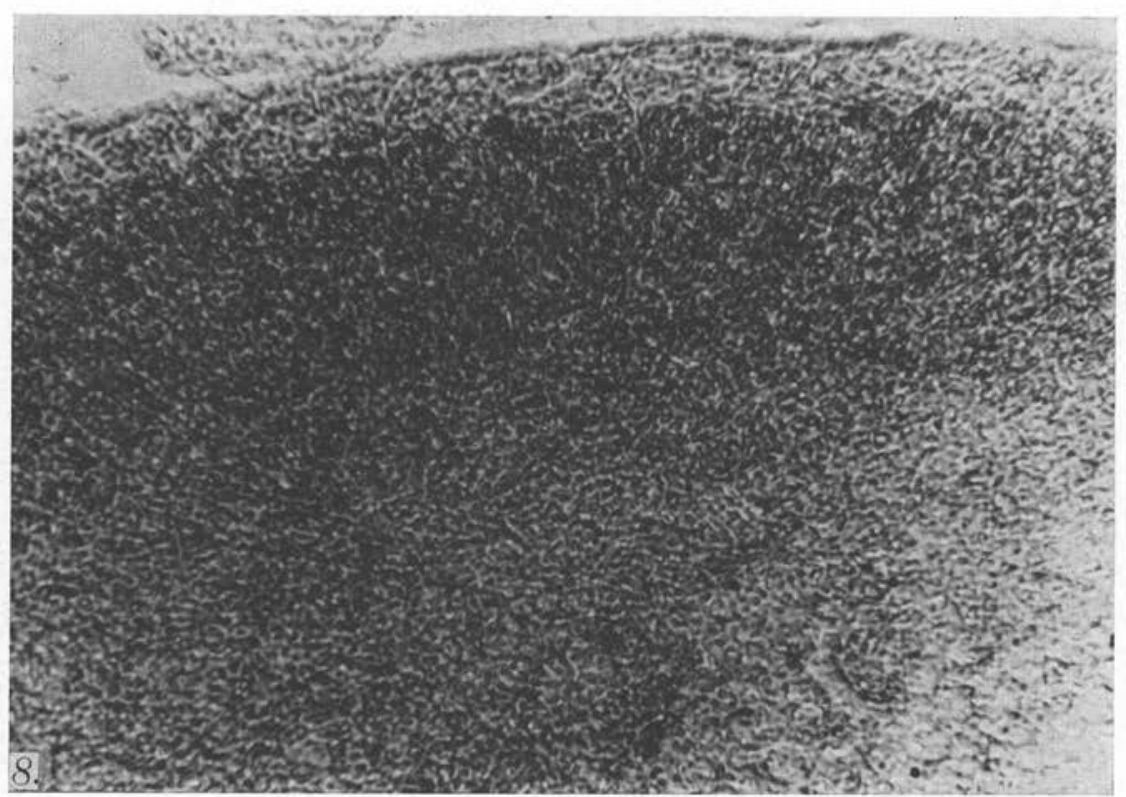

Fig. 8. - Surrénale. Animal traité avec de l'extrait ascaridien aqueux total. L'activité de la phosphatase acide est accentuée dans la zone fasciculaire et très faible dans la zone glomérulaire. Méthode Gomori. Oc. : 15 x, Ob. : Lupe 4,5/30 
Phosphatase acide. - La localisation topochimique de la phosphatase acide des animaux témoins est identique à celle de la phosphatase alcaline. En ce qui concerne l'activité enzymatique des surrénales des animaux traités, celle-ci présente des variations plus ou moins intenses dans la zone glomérulaire et dans la zone fasciculaire (fig. 7 et 8 ). Par contre, le nombre des cellules enzyme positives de la zone fasciculaire est considérablement augmenté et dans la substance médullaire, elle présente une activité plus intense que chez les témoins.

SuCCINODÉSHYdROGÉNASE. - Au point de vue topochimique, la succinodéshydrogénase se localise dans la zone glomérulaire et fasciculaire, tandis que la zone réticulaire ne présente pas d'activité enzymatique (fig. 9). La substance médullaire présente elle aussi une activité succinadéshydrogénasique, mais moins intense qu'au niveau de la substance corticale. C'est la zone fasciculaire qui est la plus riche en succinodéshydrogénase.

Chez les animaux traités, la localisation de l'enzyme est la même que chez les animaux témoins, mais tant du point de vue quantitatif que de l'intensité de la réaction, on remarque dans la zone glomérulaire et dans la zone fasciculaire, un accroissement considérable de l'activité enzymatique (fig. 10). L'activité enzymatique de la substance médullaire ne diffère pas de celle des animaux témoins.

\section{Discussion.}

D'après les observations histopathologiques et histochimiques des glandes surrénales des animaux traités, on peut admettre que l'extrait ascaridien aqueux total agit comme un agent non spécifique sur l'organisme. L'action de cette substance détermine l'apparition du syndrome d'adaptation générale de Selye, caractérisé par. l'hyperplasie corticale, associé à une production intense de corticostéroïdes avec des nécessités énergétiques considérables. L'activité enzymatique augmentée de la phosphatase acide et spécialement de la succinodéshydrogénase des zones glomérulaire et fasciculaire vient confirmer cette constatation. Ces deux enzymes, mais surtout la succinodéshydrogénase sont liées directement aux processus qui fournissent l'énergie dans la cellule, donc l'intensification de leur activité peut être interprétée comme un reflet de l'activité de synthèse de cette zone.

En même temps, on remarque aussi que l'extrait ascaridien aqueux a une action toxique directe, reflétée par des signes inflammatoires dans lesquels prédominent les lésions vasculaires. Ainsi, en certains cas, des lésions artérielles occlusives (obturantes) produisent des infarctus éndemiques focaux surtout dans la zone réticulaire, suivie de dégénération.

On sait que les cellules glandulaires présentent aussi en temps normal des signes évidents de dégénération cellulaire, la nécrobiose de ces cellules ayant lieu dans des conditions normales. Sous l'influence de l'extrait ascaridien, ce phénomène dépasse la limite du normal, en devenant un phénomène pathologique. L'accumulation de la substance P.A.S. positive autour et dans les parois des vaisscaux de la capsule de la surrénale 


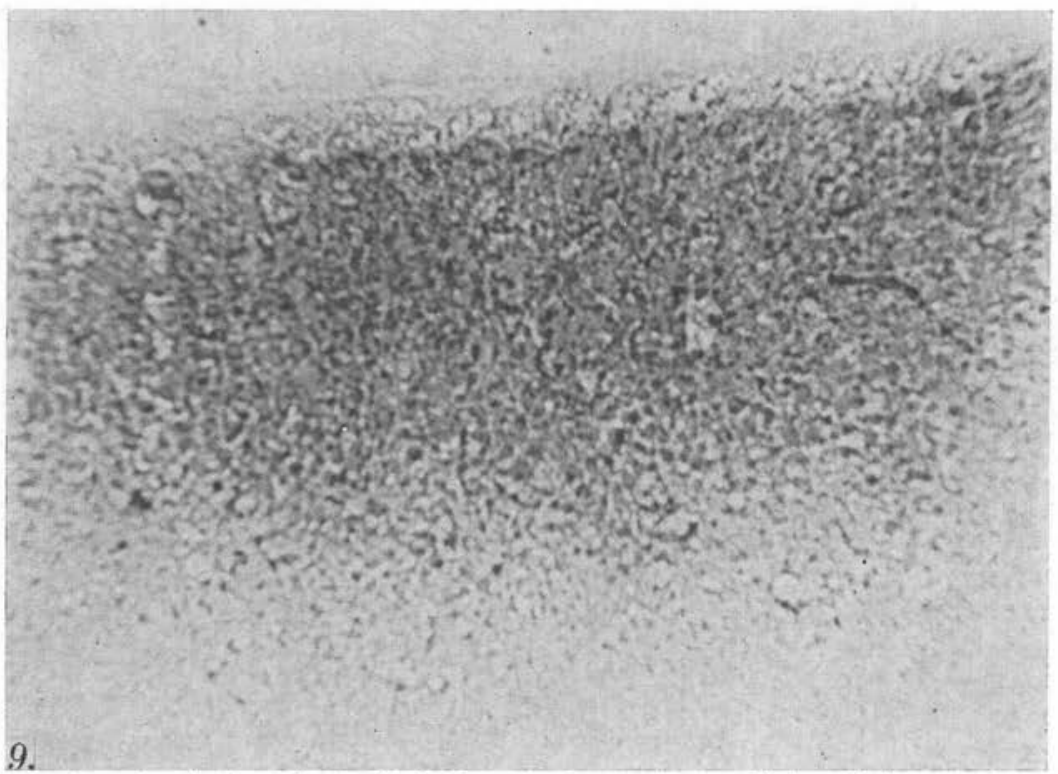

FIG. 9. - Surrénale. Animal Témoin. On peut observer une activité modérée de succinodéhidrogénase dans la zone glomérulaire et fasciculaire. Méthode Rutenburg. Oc. : Homal 6-371/2, Ob. : $8 x$

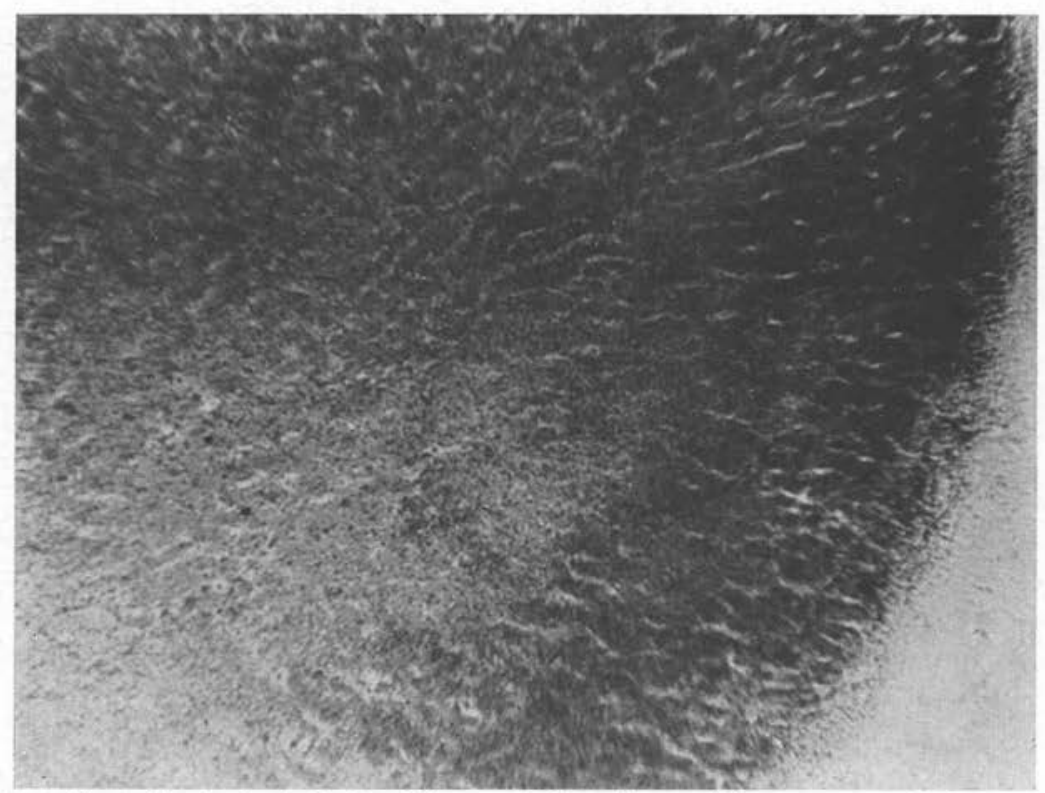

FIG. 10. - Surrénale. Animal traité avec de l'extrait ascaridien aqueux total. On peut observer une activité succinodéhidrogénasique très intense dans la zone fasciculaire. Méthode Rutenburg. Oc. : Homal 6-371/2, Ob. : 8 x 
et du cortex des animaux traités, présente des leucocytes chargés de granulations de glycogène ou à l'état libre dans les différentes zones du cortex ainsi que l'accumulation intensive du glycogène au cours de la nécrobiose de la zone réticulaire, ce qui plaide en faveur d'une action toxique directe de cette substance.

En ce qui concerne la substance médullaire des animaux traités, celle-ci ne présente pas de modifications importantes en comparaison avec celle des animaux témoins, à l'exception de l'activité de la phosphatase acide, dont la signification n'a pas été élucidée. Nos données concordent dans une certaine mesure avec celles de Schnürer, Matsura, Bach et collab. et autres $(11,8,5,4)$ qui ont trouvé chez des animaux sous stress de formaline, d'alcool de rothame (DDR) et d'autres substances toxiques ou non toxiques, une hyperplasie corticale, caractéristique du syndrome général d'adaptation. Jonek (7) et collab. ont trouvé une action augmentée dans le cortex des animaux exposés au bruit, en mettant cet accroissement de l'action enzymatique en liaison avec les nécessités énergétiques augmentées de la synthèse des hormones stéroïdes. Toute une série d'auteurs - cités par Aruin (1) ont trouvé une augmentation de l'activité de beaucoup d'enzymes, parmi lesquels celle de la succinodéshydrogénase et une augmentation de la quantité des ribonucléoprotéides dans les zones corticales de la glande surrénale dans les différentes affections. Etant donné que l'A.T.P. ainsi que la succinodéshydrogénase se localisent dans les mitochondries et qu'ils sont liés aux processus énergétiques de la cellule, on peut supposer que l'augmentation de l'action de la succinodéshydrogénase est vraiment en liaison avec la production de l'énergie nécessaire à la synthèse des hormones stéroïdes. Simultanément, l'accroissement de la quantité des ribonucléoprotéides et la disparition des lipoides de cette zone sont en rapport avec la synthèse de ces hormones (1).

Quant aux lésions artérielles, elles sont analogues à celles trouvées par Harrison (6) après administration chronique d'isoproténol, qui produit également une hypérémie corticale accompagnée d'hémorragies intracorticales suivies d'une nécrose de la zone fasciculaire et réticulaire. Les infarctus anémiques et les zones dégénérées, constatés par nous, peuvent être attribuées aux troubles trophiques qui entraînent ensuite l'oblitération des vaisseaux. D'ailleurs, Namiki (9), en étudiant les lésions vasculaires des glandes surrénales, relate des phénomènes analogues.

\section{Bibliographie}

1. Akabane (J.), Nakanishi (S.), Kohei (H.), Matsumura (R.) et Maruyama (Y.), 1964. Responses of the rat Adrenal cortex to Long-term oral administration of alcool and acetaldelyde. Med. J. Shinshu. Univ. 9/2, 659-665.

2. ARUIN (L. I.), 1966. - Morfologhiceskie kriterii sostoiania functionalnoi aktivnosti copî nadpocecinocov, 8, 9-14.

3. Babonits (M.), WiÉner (F.), FaZAKas (B.), 1965. - Modification histologique et histochimique dans les organes des animaux traités avec un extrait ascaridien. Volume commémoratif du $\mathrm{xx}^{e}$ anniversaire de l'I.M.F. Tg-Mures, 15. 
4. - , - , - 1964. - Influence des extraits ascaridiens sur les ganglions lymphatiques. Arch. Roum. Path. Exp. Microbiol, 4, 861-868.

5. BACH (I.), DAVID (G.), KeRÉLYI (N.), 1957. - A Rothane/DDD/hatása patkányok mellékvesekérgének funkciojára és szöveti képére. Orvosi hetilap, 31, 850-85.1.

6. - , 1959. - A mellékvesekéreg müködé séröl és betegségeirôl. Orvosi hetilap, 21, 741752.

7. Harrison (R. G.), Beown (K.-N.), 1964. - Suprarenal cortical degeneration following Isoproterenol administration in the rat. Endocrinology, 75/2, 173-178.

8. Jonek (J.), Stanosek (J.), Krauze (M.) et WaclawezyK (H.), 1965. - Histochemische Untersuchungen über das Verhalten einiger Enzyme in Nebennieren bei Meerschwein nach chronischer Larmeinwirkung. Z. Mikr. Anat. Forsch. 73/2, 174-186.

9. NAmiki (H.), 1964. - Vascular lesions of the Adrenal Gland. Acta Path. Jap. 14/4, 435443.

10. Rutenburg (A. M.), Wolman (M.) et Seligman (A. M.), 1954. - Ein Histochemische metoden von Walter Sipp. Lieferung II.

11. SCHNüreR (L.), 1963. - Maternal and fœtal responses to chronic stress in pregnancy. A study in albino Rats. Acta Endocr. suppl., 80-96. 